\title{
Controle em pré semeadura da cultura de soja de algodão voluntário tolerante ao glyphosate e amônio glufosinate e de Eleusine indica
}

\author{
Control in soybean pre plant of volunteer glyphosate and ammonium \\ glufosinate tolerant cotton and Eleusine indica
}

\section{Guilherme Barbosa Minozzi ${ }^{1 *}$, Pedro Jacob Christoffoleti ${ }^{2}$, Patricia Andrea Monquero ${ }^{3}$, Luiz Henrique Saes Zobiole ${ }^{1}$, Gabriel Roher Pereira ${ }^{1}$, Lucas Duck ${ }^{4}$}

Resumo - O objetivo do trabalho foi avaliar a eficácia de herbicidas aplicados em pré-emergência, em diferentes doses e épocas, antes da semeadura de soja sob condição de déficit hídrico, para o controle de plantas voluntárias de algodão, tolerante aos herbicidas glyphosate e amônio glufosinate, e Eleusine indica. O ensaio foi conduzido no município de Formosa do Rio Preto/BA, durante a safra 2015/16, utilizando o delineamento experimental de blocos ao acaso, com quatro repetições e dez tratamentos, sendo: glyphosate + diclosulam $\left(960+25,2 \mathrm{~g} \mathrm{ha}^{-1}\right.$ e.a./i.a. $)$ e glyphosate + diclosulam $\left(960+35 \mathrm{~g} \mathrm{ha}^{-1}\right.$ e.a./i.a.), aplicados aos 50, 20 e 0 dias antes da semeadura (DAS), glyphosate + flumioxazin $\left(960+50 \mathrm{~g} \mathrm{ha}^{-1}\right.$ e.a./i.a. $)$, glyphosate + sulfentrazone $\left(960+150 \mathrm{~g} \mathrm{ha}^{-1}\right.$ e.a./i.a. $)$ e glyphosate + sulfentrazone $\left(960+200 \mathrm{~g} \mathrm{ha}^{-1}\right.$ e.a./i.a.) aplicados 0 DAS, além de um tratamento controle. Avaliações visuais de controle foram efetuadas aos 7 e 0 DAS, aos 14 e 45 dias depois da semeadura (DDS) e a produtividade da soja estimada. A aplicação do herbicida diclosulam é eficaz no controle de algodão voluntário, quando aplicado 50, 20 e 0 DAS, nas doses de 25,2 e $35 \mathrm{~g} \mathrm{ha}^{-1}$ i.a. O tratamento sulfentrazone é eficaz, quando aplicado 0 DAS nas doses de 150 e $200 \mathrm{~g} \mathrm{ha}^{-1}$ i.a., enquanto o herbicida flumioxazin não é eficaz. No controle de Eleusine indica o diclosulam foi o melhor tratamento quando aplicado 0 DAS a 25,2 e $35 \mathrm{~g} \mathrm{ha}^{-1}$ i.a. A produtividade foi considerada igual entre tratamentos.

Palavras-chave: algodão tiguera, diclosulam, flumioxazin, pré emergentes, sulfentrazone

Abstract - The objective of the research was to determine the efficacy of pre-emergence-applied herbicides sprayed at different rates and times before soybean planting under water deficit conditions, to control volunteer cotton plants tolerant to glyphosate and ammonium glufosinate and Eleusine indica. The trial was conducted on Formosa do Rio Preto/BA, during the 2015/16 growing season, in a randomized complete block design, with four replications and ten treatments, which included: glyphosate + diclosulam $960+25,2 \mathrm{~g} \mathrm{ha}^{-1}$ a.i., glyphosate + diclosulam $960+35 \mathrm{~g} \mathrm{ha}^{-1}$ a.i. sprayed 50, 20 and 0 days before soybean planting (DBP), glyphosate + flumioxazin $960+50 \mathrm{~g} \mathrm{ha}^{-1}$ a.i., glyphosate + sulfentrazone $960+150 \mathrm{~g} \mathrm{ha}^{-1}$ a.i., glyphosate + sulfentrazone $960+200 \mathrm{~g} \mathrm{ha}^{-1}$ a.i. sprayed 0 DBP and an untreated treatment. Assessments of percentage visual control were performed

Recebido: Maio 18, 2017. Aceito: Setembro 05, 2017.

${ }^{1}$ Dow AgroSciences Industrial Ltda, Av. Nações Unidas, 14171, CEP 04794-000, São Paulo, SP, Brasil.

E-mail: gbminozzi@dow.com; lszobiole@dow.com; grpereira@dow.com

2 Departamento de Produção Vegetal, Escola Superior de Agricultura "Luiz de Queiroz" - ESALQ, Universidade de São Paulo - USP, Piracicaba, SP, País. E-mail: pjchrist@esaIq.usp.br

${ }^{3}$ Centro de Ciências Agrárias, Universidade Federal de São Carlos - UFSCar, Araras, SP, Brasil.

E-mail: pamonque@cca.ufscar.br

${ }^{4}$ Faculdade Arnaldo Horácio Ferreira - FAAHF, Luis Eduardo Magalhães, BA, Brasil.

E-mail: lucasduck_ld@hotmail.com 
7 and 0 DBP and 14 and 45 days after soybean planting (DAP) and soybean grain yield was measured. Diclosulam at 25,2 and $35 \mathrm{~g} \mathrm{ha}^{-1}$ a.i. controlled volunteer cotton plants when sprayed 50, 20 and 0 DBP. Sulfentrazone sprayed 0 DBP at 150 and $200 \mathrm{~g} \mathrm{ha}^{-1}$ a.i. controlled volunteer cotton while flumioxazin did not provide adequate control. Diclosulam at 25,2 to $35 \mathrm{~g} \mathrm{ha}^{-1}$ a.i. was the best treatment to control Eleusine indica when sprayed 0 DBP. Soybean yield was considered equal between treatments.

Keywords: volunteer cotton, diclosulam, flumioxazin, pre-emergence, sulfentrazone

\section{Introdução}

O cultivo do algodoeiro (Gossypium hirsutum) é uma das mais importantes e rentáveis atividades do agronegócio brasileiro, tendo em vista a grande demanda mundial por fibras naturais e óleo vegetal (Yamashita et al., 2008). Dentre as maiores regiões produtoras do país destaca-se o oeste da Bahia, com um crescimento extraordinário em área cultivada nas décadas de 80 e 90 Mendonça (2006). No Brasil, durante a safra 2015/16, foi cultivada área de 954,7 mil hectares, sendo que no estado da Bahia, segundo maior produtor nacional, á área plantada foi de 235,2 mil hectares (CONAB, 2016). No entanto, a boa prática agrícola, bem como a intensificação dos sistemas de produção têm levado a maioria dos produtores da região à sucessão de cultivo de algodão, seguido da cultura de soja (Glycine max). Assim, um dos entraves encontrados neste sistema de produção é o controle das plantas voluntárias de algodão, após a sua colheita, em pré-semeadura da soja.

O cultivo de algodoeiros resistentes ao glyphosate com a sucessão da cultura de soja, também resistente a este herbicida, tem sido cada vez mais comum no cerrado brasileiro. Consequentemente, o controle de plantas voluntárias de algodão (tiguera de algodão) é ineficiente pelo herbicida glyphosate (Ferreira et al., 2015). O curto período de tempo entre a colheita do algodoeiro e a semeadura da safra de verão de soja, dificulta um manejo efetivo das plantas daninhas, já que os herbicidas adotados podem interferir nas culturas plantadas em sucessão.

Pesquisadores enfatizam a importância de realizar o manejo adequado do algodão voluntário dentro da cultura da soja, visto que essas plantas remanescentes são fontes de disseminação do bicudo do algodoeiro (Anthonomus grandis), a principal praga da cultura (York et al., 2004).

Outra dificuldade encontrada pelo produtor de algodão no oeste da Bahia é o Eleusine indica (capim-pé-de-galinha), sendo uma planta daninha de infestação comum nos sistemas de produção, que envolvem as culturas de algodão e soja em sucessão, sendo considerado planta infestante em mais de 50 culturas no mundo, incluindo o Brasil (Radosevich et al., 1997). De acordo com Vidal et al. (2006), o capim-pé-de-galinha é mais adaptado a solos compactados, com baixa fertilidade, e elevada acidez, quando comparado a outras espécies, cenário muito comum também na região do Oeste Baiano.

Uma das grandes dificuldades práticas de manejo de plantas voluntárias e de capim-pé-de-galinha, é o controle durante os períodos de déficit hídrico que ocorre entre os cultivos das duas culturas. Poucas são as possibilidades, e pouco se conhece sobre o comportamento dos herbicidas aplicados neste período, principalmente aqueles aplicados em condições de pré-emergência, e em pré-semeadura da cultura de soja. Esta prática pode ser de grande utilidade para o produtor, não apenas para solucionar a problemática das plantas voluntárias de algodão, mas também pode auxiliar na dessecação, diversificando o sistema, e assim evitando a seleção de plantas daninhas resistentes, e de difícil controle.

Assim, o objetivo desta pesquisa foi avaliar a eficácia de herbicidas aplicados em condições de pré-emergência, associados ao glyphosate, em diferentes épocas que antecedem a semeadura de soja, com doses variáveis, no controle de novos fluxos de emergência de algodão voluntário 
tolerante aos herbicidas glyphosate e amônio glufosinate, e de capim-pé-de-galinha, em ambiente de produção da região oeste da Bahia.

\section{Material e métodos}

O ensaio foi conduzido em condição de campo no município de Formosa do Rio Preto, localizado na região oeste do estado da Bahia, durante o período de 08/10/2015 a 25/03/2016. As coordenadas geográficas da área são Latitude: $11^{\circ} 22^{\prime} 13^{\prime \prime}$ Sul, e Longitude: 46 10' 14" Oeste.

A análise física do solo resultou em $70 \%$ de areia, $10 \%$ de silte e $20 \%$ de argila. O clima predominante na região, de acordo com a classificação de Köppen (1948), é o do tipo Aw Tropical sub úmido com chuvas de verão, e período seco bem definido no inverno, e vegetação predominante de cerrado. Os dados de precipitação pluvial, coletados durante a condução do experimento, estão apresentados na Figura 1.

A semeadura da soja ocorreu em 27/11/2015, em sistema de plantio direto, utilizando-se a variedade Monsoy 8349 IPRO. A adubação foi realizada no sulco de semeadura, na dose de $250 \mathrm{~kg} \mathrm{ha}^{-1}$ da formulação contendo $20 \%$ de $\mathrm{K}_{2} \mathrm{O}$ e $20 \%$ de $\mathrm{P}_{2} \mathrm{O}_{5}$. Os demais tratos culturais foram realizados de acordo com a necessidade da cultura seguindo recomendações da EMBRAPA (2011). No local onde o ensaio foi instalado, o solo foi previamente cultivado com algodão tolerante aos herbicidas glyphosate e amônio glufosinate, que serão abreviados da seguinte forma ao longo do texto (GLY/GLU TOL).

O delineamento experimental adotado foi de blocos ao acaso, com quatro repetições, sendo cada parcela experimental de $18 \mathrm{~m}^{2}$ com 6 linhas de soja, espaçadas de $50 \mathrm{~cm}$ entre linhas por $6 \mathrm{~m}$ de comprimento, e com densidade de 10 plantas por metro linear. Foram avaliados 10 tratamentos, aplicados em diferentes épocas anterior a semeadura da soja, utilizando volume de calda equivalente a $100 \mathrm{~L} \mathrm{ha}^{-1}$ (Tabela 1).

Os ingredientes ativos dos herbicidas utilizados para compor os tratamentos foram glyphosate (Glizmax, 480 g e.a. L $^{-1}$, SL, Dow AgroSciences Industrial Ltda), diclosulam (Spider $840 \mathrm{WG}$, 840 g i.a. $\mathrm{Kg}^{-1}$, WG, Dow AgroSciences Industrial

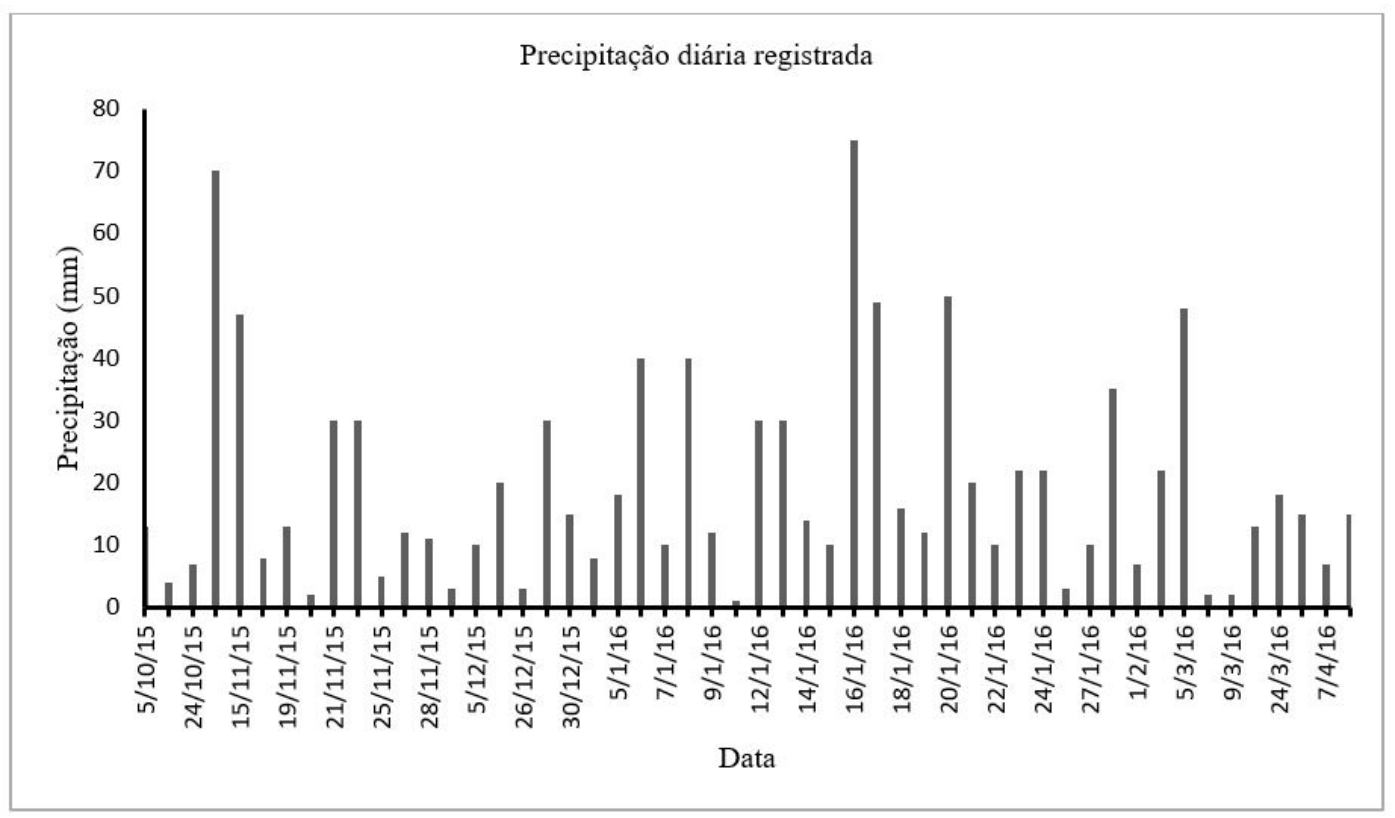

Figura 1. Precipitação diária registrada no período de 05 de outubro de 2015 a 10 de abril de 2016 durante condução do ensaio no município de Formosa do Rio Preto/BA. 
Ltda), sulfentrazone (Boral $500 \mathrm{SC}, 500 \mathrm{~g}$ i.a. $\mathrm{L}^{-1}$, SC, FMC Química do Brasil LTDA), flumioxazin (Flumyzin 500, 500 g i.a. $\mathrm{Kg}^{-1}$, WP, Sumitomo Chemical do Brasil Representações LTDA), e paraquat (Gramoxone 200, 200 g i.a. $\mathrm{L}^{-1}$, SL, Syngenta Proteção de Cultivos Ltda).

As aplicações dos tratamentos foram realizadas após a colheita do algodão (entre safra), período caraterizado por deficiência hídrica severa na região. Assim, a primeira aplicação foi realizada no dia 08 de outubro de 2015, 50 dias antes da semeadura da soja (50 DAS), e a segunda no dia 7 de Novembro de 2015, aos 20 DAS, e no dia da semeadura (0 DAS). As aplicações dos tratamentos foram efetuadas com pulverizador costal pressurizado a $\mathrm{CO}_{2}$, calibrado para um volume de calda equivalente a $100 \mathrm{~L} \mathrm{ha}^{-1}$, através de 6 pontas de aplicação do tipo AIXR110015.
As condições de aplicação dos herbicidas podem ser observadas na Tabela 2.

Imediatamente antes da aplicação dos herbicidas em condições de pré-emergência, aos 0 DAS, o herbicida paraquat foi aplicado na dose de $400 \mathrm{~g} \mathrm{ha}^{-1}$ i.a., visando garantir a morte de todas as plantas daninhas emergidas nas parcelas, não houve necessidade de realizar essa aplicação aos 50 e 20 DAS pois não haviam plantas emergidas nas parcelas devido ao longo período sem chuvas.

$\mathrm{Na}$ área experimental havia, além da presença de voluntárias de algodão, a infestação de capim-pé-de-galinha, e, portanto, também se avaliou a eficácia de controle dos tratamentos sobre esta planta daninha.

Como os tratamentos começaram a ser aplicados durante a época seca, ou seja, sem presença do capim-pé-de-galinha e plantas voluntárias de algodão emergidas, as avaliações ocorreram apenas após a emergência das mesmas nas testemunhas,

Tabela 1. Condições climáticas registradas durante as aplicações dos tratamentos efetuadas aos 50DAS, 20DAS e no dia da semeadura (0DAS).

\begin{tabular}{cccc}
\hline \multirow{2}{*}{ Dados climáticos no momento das aplicações } & \multicolumn{3}{c}{ Aplicação } \\
\cline { 2 - 4 } & 50DAS & 20DAS & 0DAS \\
\hline Temperatura mínima $\left({ }^{\circ} \mathrm{C}\right)$ & 27 & 27 & 28 \\
Temperatura máxima $\left({ }^{\circ} \mathrm{C}\right)$ & 29 & 30 & 30 \\
Velocidade do vento mínima $\left(\mathrm{Km} \mathrm{h}^{-1}\right)$ & 2 & 4 & 3 \\
Velocidade do vento máxima $\left(\mathrm{Km} \mathrm{h}^{-1}\right)$ & 4 & 6 & 5 \\
Direção do vento & Oeste & Oeste & Oeste \\
Umidade relativa do ar mínima $(\%)$ & 40 & 50 & 50 \\
Umidade relativa do ar máxima $(\%)$ & 45 & 55 & 60 \\
Nebulosidade $(\%)$ & 0 & 10 & 50 \\
\hline
\end{tabular}

Tabela 2. Tratamentos herbicidas com respectivos ingredientes ativos, doses e momento de aplicação.

\begin{tabular}{|c|c|}
\hline Tratamentos e doses (g ha ${ }^{-1}$ e.a. ou i.a.) & $\begin{array}{c}\text { Época de aplicação dos tratamentos em dias antes da } \\
\text { semeadura (DAS) }\end{array}$ \\
\hline T1. Glyphosate $(960)+$ diclosulam $(25,2)$ & 50 \\
\hline T2. Glyphosate (960) + diclosulam (35) & 50 \\
\hline T3. Glyphosate $(960)+$ diclosulam $(25,2)$ & 20 \\
\hline T4. Glyphosate (960) + diclosulam (35) & 20 \\
\hline T5. Glyphosate $(960)+$ diclosulam $(25,2)$ & 0 \\
\hline T6. Glyphosate (960) + diclosulam (35) & 0 \\
\hline T7. Glyphosate (960) + flumioxazin (50) & 0 \\
\hline T8. Glyphosate $(960)+$ sulfentrazone $(150)$ & 0 \\
\hline T9. Glyphosate (960) + sulfentrazone (200) & 0 \\
\hline T10. Controle sem aplicação de herbicida & - \\
\hline
\end{tabular}

e.a. $=$ equivalente ácido (glyphosate) e i.a. $=$ ingrediente ativo (diclosulam). 
dessa forma foi aplicado o método do quadrado inventário (Braun-Blanquet, 1979), para identificação e quantificação das plantas daninhas na área. Assim um quadrado de $1,0 \times 1,0 \mathrm{~m}$ foi lançado 4 vezes aleatoriamente nas parcelas controle sem aplicação de herbicida, amostrando área de $4 \mathrm{~m}^{2}$ por repetição, dessa forma foi obtido o nível de infestação, que apresentou densidade média de 5 plantas voluntárias de algodão por metro quadrado, e 7 plantas de capim-pé-de-galinha por metro quadrado. As eficácias de controle das plantas daninhas foram avaliadas aos 7 DAS, 0 DAS, 14 dias depois da semeadura (DDS) e 45 DDS, utilizando a escala de avaliação visual, onde zero representa ausência de controle, e 100 morte da planta (SBCPD, 1995).

Aos 45 DDS as plantas voluntárias de algodão foram removidas manualmente de todas as parcelas, inclusive do tratamento controle, visando a não proliferação do inseto bicudo do algodoeiro (Anthonomus grandis) na área, assim a interferência com a cultura da soja se deu até essa data.

A produtividade de grãos de soja foi estimada por meio de colheita manual das plantas presentes em 3 metros lineares das 4 linhas centrais de cada parcela, sendo a umidade do produto colhido corrigido para $13 \%$, sendo os resultados apresentados em $\mathrm{kg} \mathrm{ha}^{-1}$.

Todos os dados foram submetidos a análise de variância, e quando significativos o teste de Tukey ao nível de $5 \%$ de probabilidade foi adotado para comparação entre as médias.

\section{Resultados e discussão}

Os tratamentos aplicados aos 50 e 20 DAS controlaram mais de $90 \%$ da emergência de plantas de algodão voluntário nas avaliações efetuadas aos 7 e 0 DAS, permitindo a semeadura da soja sem a presença do algodão voluntário, ou seja, na condição do ensaio os tratamentos mencionados não necessitariam de uma aplicação de dessecação, o que facilitaria o manejo do sistema (Tabela 3). Na avaliação efetuada aos 0 DAS foi atribuída nota de $100 \%$ de controle para todos os tratamentos aplicados aos 0 DAS, pois o herbicida paraquat utilizado em todos os tratamentos, como mencionado nos materiais e métodos, foi efetivo no controle das plantas já emergidas de algodão.

Aos 14 DDS todos os tratamentos com diclosulam aplicados na dose de $35 \mathrm{~g} \mathrm{ha}^{-1}$ i.a. promoveram controle superior a $80 \%$ do algodão

Tabela 3. Porcentagem visual de controle do algodão voluntário (GLY/GLU TOL) em diferentes épocas.

\begin{tabular}{|c|c|c|c|c|c|c|c|c|}
\hline \multirow{3}{*}{$\frac{\text { Tratamentos (g ha }}{\text {-1 e.a. ou i.a.) }}$} & \multirow{3}{*}{$\begin{array}{l}\begin{array}{r}\text { Época de } \\
\text { aplicação }\end{array} \\
50 \text { DAS }\end{array}$} & \multicolumn{7}{|c|}{ \% visual de controle de algodão voluntário } \\
\hline & & \multirow{2}{*}{$\begin{array}{l}\text { 7DAS } \\
100\end{array}$} & \multicolumn{2}{|c|}{ ODAS } & \multicolumn{2}{|c|}{ 14DDS } & \multicolumn{2}{|c|}{ 45DDS } \\
\hline & & & 95,3 & $\mathrm{ab}$ & 77,0 & $\mathrm{bc}$ & 68,6 & $\mathrm{ab}$ \\
\hline T2. Glyphosate (960) + diclosulam (35) & 50 DAS & 100 & 95,3 & $\mathrm{ab}$ & 80,7 & $a b$ & 75,7 & $\mathrm{a}$ \\
\hline T3. Glyphosate $(960)+$ diclosulam $(25,2)$ & 20 DAS & 100 & 94 & $\mathrm{~b}$ & 68,7 & $\mathrm{~cd}$ & 55,3 & $\mathrm{bc}$ \\
\hline T4. Glyphosate (960) + diclosulam (35) & 20 DAS & 100 & 96,5 & $\mathrm{ab}$ & 83,3 & $a b$ & 68,9 & $\mathrm{ab}$ \\
\hline T5. Glyphosate $(960)+$ diclosulam $(25,2)$ & 0 DAS & --- & 100 & $\mathrm{a}$ & 84,5 & $a b$ & 81,9 & $\mathrm{a}$ \\
\hline T6. Glyphosate (960) + diclosulam (35) & 0 DAS & --- & 100 & $\mathrm{a}$ & 85,1 & $a b$ & 79,3 & $\mathrm{a}$ \\
\hline T7. Glyphosate (960) + flumioxazin (50) & 0 DAS & --- & 100 & $\mathrm{a}$ & 60,4 & $\mathrm{~d}$ & 42,1 & $\mathrm{c}$ \\
\hline T8. Glyphosate (960) + sulfentrazone (150) & 0 DAS & --- & 100 & $\mathrm{a}$ & 85,1 & $a b$ & 79,3 & $\mathrm{a}$ \\
\hline T9. Glyphosate (960) + sulfentrazone (200) & 0 DAS & --- & 100 & $\mathrm{a}$ & 90,0 & $\mathrm{a}$ & 81,9 & $\mathrm{a}$ \\
\hline T10. Controle sem herbicida & - & --- & 0 & $\mathrm{c}$ & 0 & $\mathrm{e}$ & 0 & d \\
\hline$F$ & & --- & $989,53 *$ & & $163,69 *$ & & $67,94 *$ & \\
\hline $\mathrm{CV}(\%)$ & & --- & 2,39 & & 5,82 & & 9,84 & \\
\hline DMS & & --- & 5,01 & & 10,12 & & 15,15 & \\
\hline
\end{tabular}

Médias seguidas da mesma letra na coluna não diferem estatisticamente entre si através do teste de Tukey ao nível de $5 \%$ de probabilidade. * Indica que o valor de $\mathrm{F}$ obtido através da análise de variância é significativo, ou seja, ao menos uma das médias de tratamentos difere das demais. 
voluntário, independente do momento em que foram aplicados, enquanto que os tratamentos aplicados na dose de 25,2 $\mathrm{g} \mathrm{ha}^{-1}$ i.a. somente promoveram esse mesmo nível de controle quando aplicado 0 DAS (Tabela 3). Ainda aos 14 DDS, o tratamento com flumioxazin apresentou controle de $60,4 \%$, enquanto os tratamentos com sulfentrazone proporcionaram controle acima de $80 \%$. Através destes resultados é possível inferir que o diclosulam, mesmo quando aplicado em condição de déficit hídrico (50 DAS) persistiu na solução do solo em concentrações suficientes para o controle do algodão voluntário (Tabela 3).

$\mathrm{Na}$ última avaliação, aos $45 \mathrm{DDS}$, próximo ao momento em que a área foliar da soja cobria totalmente a superfície do solo, observa se que os tratamentos com diclosulam a $35 \mathrm{~g} \mathrm{ha}^{-1}$ i.a. aplicados aos 50, 20 e 0 DAS foram considerados estatisticamente iguais e promoveram controle superior a 70\% do algodão voluntário, sugerindo que o produto pode ser aplicado em período de déficit hídrico, até 50 DAS da cultura da soja, e assim reduzir o número de plantas de algodão emergidas durante o desenvolvimento da soja. É possível afirmar que com este resultado, na prática, reduz a necessidade de aplicação de herbicidas dessecantes em pré-semeadura, e também pode-se reduzir o número de aplicações de herbicidas de pós-emergência da soja para o controle de algodão voluntário.

Já a dose de 25,2 $\mathrm{g} \mathrm{ha}^{-1}$ i.a. de diclosulam também foi efetiva quando aplicada em período de déficit hídrico, porém apresentou maior variabilidade em função do momento da aplicação e da avaliação, com níveis de controle de 55,3\% para aplicação efetuada a 20 DAS e $68,6 \%$ quando aplicado 50DAS. Entretanto, quando essa dose de diclosulam é utilizada mais próximo a semeadura da soja, o controle obtido aos 45 DDS foi de $81,9 \%$, sendo considerado estatisticamente igual a dose de $35 \mathrm{~g} \mathrm{ha}^{-1}$ i.a. aplicada na mesma data.

Dessa forma para o herbicida diclosulam, quando o mesmo é aplicado aos 50 ou 20 DAS, ambas as doses testadas promovem controle efetivo do algodão voluntário até os 0 DAS, permitindo que o plantio da soja seja realizado sem a presença dessa planta daninha, porém para que o controle permaneça elevado até 45 DDS a dose de $35 \mathrm{~g} \mathrm{ha}^{-1}$ i.a. deve ser aplicada a 50, $20 \mathrm{ou}$ 0 DAS, enquanto a dose de 25,2 $\mathrm{g} \mathrm{ha}^{-1}$ i.a deve ser aplicada no dia da semeadura.

O tratamento envolvendo flumioxazin não foi efetivo para controle do algodão voluntário, apresentando controle de $42,1 \%$, enquanto os tratamentos com sulfentrazone foram efetivos e considerados estatisticamente iguais aos tratamentos com diclosulam aplicados no dia da semeadura, proporcionando nível de controle em torno de $80 \%$.

Controle eficaz, maior que $80 \%$ em algodão voluntário, foi verificado utilizando o herbicida S-metolachor + metribuzin na dose de 2,33 $\mathrm{L} \mathrm{ha}^{-1}$ (Morgan et al., (2011). Controle de 100\% em algodão voluntário foi observado utilizando o herbicida flumetsulam ( $45 \mathrm{~g} \mathrm{ha}^{-1}$ i.a.) e imazaquin (137 $\mathrm{g} \mathrm{ha}^{-1}$ i.a.) e controle de $95 \%$ foi observado quando utilizado metribuzin + chlorimurom $\left(360+60 \mathrm{~g} \mathrm{ha}^{-1}\right.$ i.a. $)$ e sulfentrazone + chlorimuron $\left(167+34 \mathrm{~g} \mathrm{ha}^{-1}\right.$ i.a. $)$, porém quando o herbicida metribuzin foi utilizado de forma isolado, os autores observaram controle de 83\% York et al. (2004).

Nas avaliações realizadas aos 7 e 0 DAS, observou-se que os tratamentos com diclosulam nas doses de 25,2 e $35 \mathrm{~g} \mathrm{ha}^{-1}$ i.a., aplicados 50 e 20 DAS, apresentaram controle superior a $90 \%$ do capim-pé-de-galinha, demonstrando o efeito residual fornecido pelo diclosulam (Tabela 4). Os tratamentos aplicados $0 \mathrm{DAS}$, ainda não haviam sido aplicados na ocasião da avaliação aos 7 DAS, portanto não foram atribuidas notas, enquanto na avaliação realizada aos 0 DAS os tratamentos aplicados nessa data receberam a nota de $100 \%$ de controle, visto que o herbicida paraquat foi eficaz no controle dessa planta daninha.

Aos 14 DDS é possível observar que os tratamentos mais eficientes foram os tratamentos com diclosulam aplicados aos 0 DAS, que apresentaram controle 
Tabela 4. Porcentagem visual de controle do capim-pé-de-galinha em diferentes épocas.

\begin{tabular}{|c|c|c|c|c|c|c|c|c|}
\hline \multirow{2}{*}{ Tratamentos (g ha-1 e.a. ou i.a.) } & \multirow{2}{*}{$\begin{array}{l}\text { Época de } \\
\text { aplicação }\end{array}$} & \multicolumn{7}{|c|}{$\%$ visual de controle de capim-pé-de-galinha } \\
\hline & & 7DAS & ODAS & & 14D & & 45D & \\
\hline T1. Glyphosate $(960)+$ diclosulam $(25,2)$ & 50 DAS & 100,0 & 94,8 & $\mathrm{a}$ & 58,3 & abcd & 56,1 & bcde \\
\hline T2. Glyphosate (960) + diclosulam (35) & 50 DAS & 100,0 & 94,8 & a & 65,6 & $a b c$ & 64,8 & $a b c$ \\
\hline T3. Glyphosate $(960)+$ diclosulam $(25,2)$ & 20 DAS & 98,7 & 94,0 & $\mathrm{a}$ & 52,8 & bcd & 52,8 & cde \\
\hline T4. Glyphosate (960) + diclosulam (35) & 20 DAS & 99,3 & 94,0 & $\mathrm{a}$ & 63,2 & $a b c$ & 61,6 & abcd \\
\hline T5. Glyphosate $(960)+$ diclosulam $(25,2)$ & 0 DAS & --- & 100,0 & a & 71,9 & $\mathrm{ab}$ & 74,3 & $\mathrm{ab}$ \\
\hline T6. Glyphosate (960) + diclosulam (35) & 0 DAS & --- & 100,0 & a & 79,3 & a & 76,9 & a \\
\hline T7. Glyphosate (960) + flumioxazin (50) & 0 DAS & --- & 100,0 & a & 63,8 & $a b c$ & 58,7 & abcd \\
\hline T8. Glyphosate (960) + sulfentrazone (150) & 0 DAS & --- & 100,0 & $\mathrm{a}$ & 35,9 & $d$ & 37,8 & e \\
\hline T9. Glyphosate (960) + sulfentrazone (200) & 0 DAS & --- & 100,0 & $\mathrm{a}$ & 44,8 & $\mathrm{~cd}$ & 43,6 & de \\
\hline T10. Controle sem a aplicação de herbicida & & --- & 0,0 & $\mathrm{~b}$ & 0,0 & $\mathrm{e}$ & 0,0 & $\mathrm{f}$ \\
\hline$F$ & & --- & $345,52 *$ & & $18,97 *$ & & $30,04 *$ & \\
\hline CV (\%) & & --- & 3,80 & & 19,42 & & 15,34 & \\
\hline DMS & & --- & 8,10 & & 25,30 & & 19,64 & \\
\hline
\end{tabular}

Médias seguidas da mesma letra na coluna não diferem estatisticamente entre si através do teste de Tukey ao nível de 5\% de probabilidade. * Indica que o valor de $\mathrm{F}$ obtido através da análise de variância é significativo, ou seja, ao menos uma das médias de tratamentos difere das demais.

acima de $70 \%$. Os tratamentos com diclosulam, em ambas as doses, aplicados aos 50 e 20 DAS apresentaram controle da ordem de 52,8 a $65,6 \%$, equiparando-se ao tratamento com flumioxazin, que apresentou eficácia de 63,8\%. Entretanto, ambos os tratamentos com sulfentrazone não foram eficazes no controle desta planta daninha, com níveis de controle de 35,9 e 44,8\% (Tabela 4).

Os tratamentos com diclosulam a $25,2 \mathrm{e} 35 \mathrm{~g} \mathrm{ha}^{-1}$ i.a. aplicados 0 DAS ainda apresentaram controle de capim-pé-de-galinha, acima de $70 \%$ aos 45 DDS da soja (Tabela 4). Já para os tratamentos aplicados 50 e 20 DAS foi possível observar uma eficiência maior na dose de $35 \mathrm{~g} \mathrm{ha}^{-1}$ i.a. de diclosulam em relação a $25,2 \mathrm{~g} \mathrm{ha}^{-1}$ i.a., porém para que o controle seja elevado até essa data a maior dose deve ser aplicada próximo a semeadura da soja. $\mathrm{O}$ tratamento com flumioxazin apresentou controle de 58,7\%, enquanto os tratamentos com sulfentrazone permaneceram fornecendo baixo controle nas duas doses, sendo $37,8 \%$ e $43,6 \%$ respectivamente.

Dessa forma para o herbicida diclosulam, o mesmo pode ser aplicado nas duas doses aos 50 ou 20 DAS para promover controle efetivo até o dia da semeadura, reduzindo a necessidade da aplicação de dessecação, porém quando se busca manter o controle elevado até os 45 DDS deve-se utilizar a dose de $35 \mathrm{~g} \mathrm{ha}^{-1}$ i.a. aplicada no dia da semeadura da soja.

Trabalhando com herbicidas pré emergentes para o controle do capim-pé-de-galinha, foi observado controle de $90 \%$ utilizando o herbicida indaziflam $\left(0,07 \mathrm{~kg} \mathrm{ha}^{-1}\right.$ i.a $)$ e de $86 \%$ utilizando oxadiazon $\left(4,48 \mathrm{~kg} \mathrm{ha}^{-1}\right.$ i.a.) (McCullough et al., 2013). Entretanto outro autor verificou controle de $74 \%$ do capim-pé-de-galinha proporcionado pelo herbicida oxadiazon $\left(4,5 \mathrm{~kg} \mathrm{ha}^{-1}\right.$ i.a.) (Johnson, 1994).

Diferentes tratamentos com pré emergentes foram testados por pesquisadores, onde verificaram $100 \%$ de controle do capim-pé-de-galinha utilizando clomazone + ethalfluralin + halosulfuron $\left(0,17+0,63+0,02 \mathrm{~kg} \mathrm{ha}^{-1}\right.$ i.a) (Brandenbergeretal.,2005) e entre 74 a $100 \%$ de controle utilizando clomazone (0,8 $\mathrm{kg} \mathrm{ha}^{-1}$ i.a.) (Scott et al., 2002).

Não houve diferença estatística significativa entre os tratamentos em relação a produtividade da cultura da soja (Tabela 5). A densidade das plantas daninhas nas parcelas pode não ter sido suficiente para promover elevada matocompetição com a cultura da soja e reduzir consequentemente a produtividade em níveis 
Tabela 5. Médias da produtividade da cultura de soja, avaliada ao final do ensaio.

\begin{tabular}{|c|c|c|}
\hline Tratamentos (g ha-1 e.a. ou i.a.) & Época de aplicação & Produtividade Kg ha ${ }^{-1}$ \\
\hline T1. Glyphosate $(960)+$ Diclosulam $(25,2)$ & $50 \mathrm{DAS}$ & 2437,6 \\
\hline T2. Glyphosate (960) + Diclosulam (35) & $50 \mathrm{DAS}$ & 2187,1 \\
\hline T3. Glyphosate $(960)+$ Diclosulam $(25,2)$ & 20 DAS & 2364,5 \\
\hline T4. Glyphosate (960) + Diclosulam (35) & 20 DAS & 2306,6 \\
\hline T5. Glyphosate $(960)+$ Diclosulam $(25,2)$ & 0 DAS & 2602,4 \\
\hline T6. Glyphosate (960) + Diclosulam (35) & 0 DAS & 2548,7 \\
\hline T7. Glyphosate (960) + Flumioxazin (50) & 0 DAS & 2540,9 \\
\hline T8. Glyphosate (960) + Sulfentrazone (150) & 0 DAS & 2424,0 \\
\hline T9. Glyphosate (960) + Sulfentrazone (200) & 0 DAS & 2351,0 \\
\hline T10. Controle sem a aplicação de herbicida & - & 1925,2 \\
\hline$F$ & & $1,705^{N S}$ \\
\hline CV $(\%)$ & & 12,90 \\
\hline DMS & & 743,31 \\
\hline
\end{tabular}

Médias seguidas da mesma letra na coluna não diferem estatisticamente entre si através do teste de Tukey ao nível de $5 \%$ de probabilidade. ${ }^{N S}$ significa que o valor de $\mathrm{F}$ obtido através da análise de variância é não significativo e portanto não há diferença entre as médias de tratamentos.

elevados. Outra hipótese é que provavelmente como as plantas de algodão voluntário foram removidas das parcelas após a última avaliação visual de controle aos 45 DDS, as diferenças de produtividade obtidas entre os tratamentos foram menores. Mesmo assim a produtividade na parcela controle foi de $1925,2 \mathrm{~kg} \mathrm{ha}^{-1}$ contra uma produtividade de $2602,4 \mathrm{~kg} \mathrm{ha}^{-1}$ referente ao tratamento $\mathrm{T} 6$, o mais produtivo, gerando uma diferença numérica de $677,2 \mathrm{~kg} \mathrm{ha}^{-1}$.

\section{Conclusões}

A aplicação do herbicida diclosulam é eficaz no controle de algodão voluntário GLY/GLU TOL até o dia da semeadura da soja quando aplicado no período de déficit hídrico, 50 ou 20 DAS, nas doses de 25,2 e $35 \mathrm{~g} \mathrm{ha}^{-1}$ i.a. Para que o controle permaneça satisfatório até 45 DDS o diclosulam deve ser aplicado aos 50, 20 ou 0 DAS a $35 \mathrm{~g} \mathrm{ha}^{-1}$ i.a. ou a $25,2 \mathrm{~g} \mathrm{ha}^{-1}$ i.a. no dia da semeadura. O herbicida sulfentrazone é eficaz no controle do algodão voluntário, quando aplicado no dia da semeadura da soja nas doses de 150 e $200 \mathrm{~g} \mathrm{ha}^{-1}$ i.a., enquanto o herbicida flumioxazin não é eficaz aplicado no dia da semeadura da soja na dose de $50 \mathrm{~g} \mathrm{ha}^{-1}$ i.a.
No controle de capim-pé-de-galinha, o diclosulam é mais eficaz quando aplicado no dia da semeadura de soja nas doses de 25,2 e $35 \mathrm{~g} \mathrm{ha}^{-1}$ i.a., já os herbicidas flumioxazin e sulfentrazone não são efetivos, proporcionando controle inferior a $60 \%$ e $50 \%$ respectivamente, quando aplicados no dia da semeadura.

A aplicação do herbicida diclosulam em condição de deficiência hídrica no solo, anterior a semeadura da cultura da soja, pode ser viável, com possibilidade de reduzir a aplicação de herbicidas na operação de dessecação e pós emergência da cultura da soja.

\section{Referências}

Brandenberger, L.P.; Shrefler, J.W.; Weber III, C.L.; Talbert, R.R.; Payton, M.E.; Wells, L.K.; et al. Preemergence weed control in directseeded watermelon. Weed Technology, v.19, p.706-712, 2005.

Braun-Blanquet, J. Fitossociologia: bases para el estudio de las comunidades vegetales. Madri: H. Blume, 1979. 820p.

CONAB - Companhia Nacional de Abastecimento. Acompanhamento da safra brasileira grãos: 
safra 2015/16: décimo segundo levantamento. Brasília: CONAB, 2016. p.74-78. v. 12.

EMBRAPA - Empresa Brasileira de Pesquisa Agropecuária. Tecnologias de produção de soja: Região Central do Brasil, 2012 e 2013. Londrina: EMBRAPA, 2011. 261p.

Ferreira, A.C.B.; Bogiani, J.C.; Barbieri, A.L.; Moraes, M.C.G.; Santos, T.J.S. Controle químico de plantas voluntárias de algodão resistente ao Glyphosate na cultura da soja RR. In: Congresso Brasileiro do Algodão, 10., 2015, Foz do Iguaçu. Resumos... Brasília: ABRAPA, 2015.

Johnson, B.J. Herbicide programs for large crabgrass and goosegrass control in Kentucky bluegrass turf. HortScience, v.8, p.876-879, 1994.

Köppen, W. Climatologia: con un estudio de los climas de la tierra. México: Fondo de Cultura Econômica, 1948. 479p.

Mccullough, P.E.; Yu, J.; Barreda, D.G. Efficacy of preemergence herbicides for controlling a Dinitroaniline-resistant Goosegrass (Eleusine indica) in Georgia. Weed Technology, v.27, p.639-644, 2013.

Mendonça, J.O. O potencial de crescimento da produção de grãos do oeste da Bahia. Revista Bahia Agrícola, v.7, n.2, p.38-46, 2006.

Morgan, G.D.; Fromme, P.A.; Baumann, J.; Grichar, B.B.; Matocha, M.E.; Mott, D.A. Managing volunteer cotton in grain crops. Texas: Texas AgriLife Extension Sevice, The Department of Soil and Crop Sciences, 2011. Disponível em: <http://publications.tamu.edu/COTTON/
PUB_cotton_Managing\%20Volunteer $\% 20$ Cotton\%20in\%20Grain\%20Crops.pdf>. Acesso em: 3 abr. 2017.

Radosevich, S.R.; Ghersa, C.M.; Holt, J.S. Plantplant associations. In: Radosevich, S.R.; Ghersa, C.M.; Holt, J.S. Ecology of weeds and invasive plants: relationship to agriculture and natural resource management. 3.ed. New Jersey: John Wiley \& Sons, 1997. 454p.

SBCPD - Sociedade Brasileira da Ciência das Plantas Daninhas. Procedimentos para instalação, avaliação e análise de experimentos com herbicidas. Londrina, 1995, 42 p.

Scott, G.H.; Askew, S.D.; Wilcut, J.W. Glyphosate systems for weed control in glyphosate-tolerant cotton (Gossypium hirsutum). Weed Technology, v.16, p.191-198, 2002.

Vidal, R.A.; Portes, E.S.; Lamego, F.P.; Trezzi, M.M. Resistência de Eleusine indica aos inibidores de ACCase. Planta Daninha, v.24, p.163-171, 2006. http://dx.doi.org/10.1590/ S0100-83582006000100021.

Yamashita, O.M.; Mendonça, F.S.; Orsi, J.V.N.; Resende, D.D.; Kappes, C.; Guimarães, S.C. Efeito de doses reduzidas de oxyfluorfen em cultivares de algodoeiro. Planta Daninha, v.26, n.4, p.917-921, 2008.

York, A.C.; Stewart, A.M.; Vidrine, P.R.; Culpepper, A.S. Control of volunteer glyphosate-resistant cotton in glyphosate-resistant soybean. Weed Technology, v.18, p.532-539, 2004. 